MATHEMATICS OF COMPUTATION

Volume 80, Number 274, April 2011, Pages 1011-1018

S $0025-5718(2010) 02428-7$

Article electronically published on October 25, 2010

\title{
SHARP BOUNDS OF THE LANDAU CONSTANTS
}

\author{
CRISTINEL MORTICI
}

Abstract. The aim of this paper is to establish new bounds of the Landau constants.

\section{INTRODUCTION AND MOTIVATION}

The Landau constants defined for all positive integers $n$ by

$$
G_{n}=1+\left(\frac{1}{2}\right)^{2}+\left(\frac{1 \cdot 3}{2 \cdot 4}\right)^{2}+\left(\frac{1 \cdot 3 \cdot 5}{2 \cdot 4 \cdot 6}\right)^{2}+\cdots+\left(\frac{1 \cdot 3 \cdot \cdots \cdot(2 n-1)}{2 \cdot 4 \cdot \cdots \cdot(2 n)}\right)^{2},
$$

play an important role in some extremal problems in complex analysis and in the theory of Fourier series. More precisely, in 1913 Landau [6] proved that $G_{n}$ is the maximum of the expression $\left|\sum_{k=1}^{n} a_{k}\right|$, with respect to all functions of the form $f(z)=\sum_{k=0}^{\infty} a_{k} z^{k}$ which is analytic in the unit disk and satisfies $|f(z)|<1$, for every $|z|<1$.

In consequence, the problem of approximation of the Landau constants have attracted the attention of many authors. In particular, Landau himself studied the asymptotic behaviour of $G_{n}$ and showed that

$$
G_{n} \sim \frac{1}{\pi} \ln n
$$

then Watson [7] established the following asymptotic formula

$$
G_{n}=c_{0}+\frac{1}{\pi} \ln (n+1)-\frac{1}{4 \pi(n+1)}+O\left(\frac{1}{n^{2}}\right) \quad(n \rightarrow \infty) .
$$

Here, and in what follows, $c_{0}=\frac{1}{\pi}(\gamma+4 \ln 2)=1.06627 \ldots$, and $\gamma=0.577215 \ldots$ is the Euler-Mascheroni constant.

This problem of approximation of the Landau constants was continued in the works of Brutman [3] and Falaleev [5], who proved that for every non-negative integer $n$,

respectively

$$
1+\frac{1}{\pi} \ln (n+1)<G_{n}<1.0663+\frac{1}{\pi} \ln (n+1),
$$

$$
1.0662+\frac{1}{\pi} \ln \left(n+\frac{3}{4}\right)<G_{n}<1.0916+\frac{1}{\pi} \ln \left(n+\frac{3}{4}\right) .
$$

Received by the editor November 13, 2009 and, in revised form, February 26, 2010.

2010 Mathematics Subject Classification. Primary 41A60; Secondary 26D15.

Key words and phrases. Landau constants, approximations, inequalities, asymptotic expansions. 
We improve the upper bound in the following way, that also shows that the constant $3 / 4$ is the best possible.

Theorem 1. For every integer $n \geq 1$, we have

$$
c_{0}+\frac{1}{\pi} \ln \left(n+\frac{3}{4}\right)<G_{n}<c_{0}+\frac{1}{\pi} \ln \left(n+\frac{3}{4}+\frac{11}{192 n}\right) .
$$

Very recently, Zhao [8, Corollary 1] extended the asymptotic expansion of $G_{n}$ to

$$
G_{n}=c_{0}+\frac{1}{\pi} \ln (n+1)-\frac{1}{4 \pi(n+1)}+\frac{5}{192 \pi(n+1)^{2}}+O\left(\frac{1}{(n+1)^{3}}\right),
$$

as a direct consequence of the following double inequality

$$
\begin{aligned}
& c_{0}+\frac{1}{\pi} \ln (n+1)-\frac{1}{4 \pi(n+1)}+\frac{5}{192 \pi(n+1)^{2}}<G_{n} \\
< & c_{0}+\frac{1}{\pi} \ln (n+1)-\frac{1}{4 \pi(n+1)}+\frac{5}{192 \pi(n+1)^{2}}+\frac{3}{128 \pi(n+1)^{3}}
\end{aligned}
$$

stated in [8, Theorem 1], as the main result.

In this paper we establish the following double inequality that improves much of Zhao's results (1.1)-(1.2).

Theorem 2. For every integer $n \geq 1$, we have

$$
\begin{aligned}
c_{0}+ & \frac{1}{\pi} \ln (n+1)-\frac{1}{4 \pi(n+1)}+\frac{5}{192 \pi(n+1)^{2}} \\
+ & \frac{3}{128 \pi(n+1)^{3}}-\frac{341}{122880 \pi(n+1)^{4}}-\frac{75}{8192 \pi(n+1)^{5}}<G_{n} \\
& <c_{0}+\frac{1}{\pi} \ln (n+1)-\frac{1}{4 \pi(n+1)}+\frac{5}{192 \pi(n+1)^{2}} \\
& +\frac{3}{128 \pi(n+1)^{3}}-\frac{341}{122880 \pi(n+1)^{4}}
\end{aligned}
$$

and the following asymptotic formula holds:

$$
\begin{aligned}
G_{n} & =c_{0}+\frac{1}{\pi} \ln (n+1)-\frac{1}{4 \pi(n+1)}+\frac{5}{192 \pi(n+1)^{2}} \\
& +\frac{3}{128 \pi(n+1)^{3}}-\frac{341}{122880 \pi(n+1)^{4}}+O\left(\frac{1}{(n+1)^{5}}\right) .
\end{aligned}
$$

Another direction for developing the problem of approximation of $G_{n}$ was opened by Cvijović and Klinowski [4, Theorem 1] who gave some estimates in terms of the digamma function $\psi=\Gamma^{\prime} / \Gamma$, namely

$$
c_{0}+\frac{1}{\pi} \psi\left(n+\frac{5}{4}\right)<G_{n}<1.0725+\frac{1}{\pi} \psi\left(n+\frac{5}{4}\right)
$$

and

$$
0.9883+\frac{1}{\pi} \psi\left(n+\frac{3}{2}\right)<G_{n}<c_{0}+\frac{1}{\pi} \psi\left(n+\frac{3}{2}\right) \quad(n \geq 0) .
$$


Motivated by the work of Cvijović and Klinowski [4, Alzer [2, Theorem 1] proved the following double inequality, for every integer $n \geq 1$,

$$
c_{0}+\frac{1}{\pi} \psi(n+\alpha)<G_{n}<c_{0}+\frac{1}{\pi} \psi(n+\beta),
$$

where the constants $\alpha=5 / 4$ and $\beta=\psi^{-1}\left(\pi\left(1-c_{0}\right)\right)=1.26621 \ldots$ are the best possible.

We establish the following double inequality that improves much of the results (1.3) - (1.5) of Cvijović, Klinowski and Alzer, and also shows that the best approximation of the form

$$
G_{n} \approx c_{0}+\frac{1}{\pi} \psi(n+b) \quad(n \rightarrow \infty)
$$

is obtained for $b=5 / 4$.

Theorem 3. For every positive integer n, we have

$$
\begin{aligned}
c_{0} & +\frac{1}{\pi} \psi\left(n+\frac{5}{4}\right)+\frac{1}{64 \pi n^{2}}-\frac{3}{128 \pi n^{3}}<G_{n} \\
& <c_{0}+\frac{1}{\pi} \psi\left(n+\frac{5}{4}\right)+\frac{1}{64 \pi n^{2}}-\frac{3}{128 \pi n^{3}}+\frac{173}{8192 \pi n^{4}} .
\end{aligned}
$$

\section{The Proofs}

Our new results announced in the previous section are established using an original approach. More precisely, we use an excellent result of Alzer 1, Theorem 8 ], who proved that for every $n \geq 0$, the functions

$$
F_{n}(x)=\ln \Gamma(x+1)-\left(x+\frac{1}{2}\right) \ln x+x-\frac{1}{2} \ln 2 \pi-\sum_{i=1}^{2 n} \frac{B_{2 i}}{2 i(2 i-1) x^{2 i-1}}
$$

and

$$
H_{n}(x)=-\ln \Gamma(x+1)+\left(x+\frac{1}{2}\right) \ln x-x+\frac{1}{2} \ln 2 \pi+\sum_{i=1}^{2 n+1} \frac{B_{2 i}}{2 i(2 i-1) x^{2 i-1}}
$$

are completely monotonic ( $B_{j}$ is the $j$ th Bernoulli number). In particular, $F_{n}>0$ and $H_{n}>0$ and, consequently,

$$
s(x)<\ln \Gamma(x+1)<t(x)
$$

where

$$
s(x)=\ln \sqrt{2 \pi}+\left(x+\frac{1}{2}\right) \ln x-x+\frac{1}{12 x}-\frac{1}{360 x^{3}}+\frac{1}{1260 x^{5}}-\frac{1}{1680 x^{7}}
$$

and

$t(x)=\ln \sqrt{2 \pi}+\left(x+\frac{1}{2}\right) \ln x-x+\frac{1}{12 x}-\frac{1}{360 x^{3}}+\frac{1}{1260 x^{5}}-\frac{1}{1680 x^{7}}+\frac{1}{1188 x^{9}}$.

Then, using (2.2), we get

$$
e^{u(x)}<\frac{1}{16^{x}}\left(\frac{\Gamma(2 x+1)}{(\Gamma(x+1))^{2}}\right)^{2}<e^{v(x)},
$$

where

$$
u(x)=2 s(2 x)-4 t(x)-x \ln 16, \quad v(x)=2 t(2 x)-4 s(x)-x \ln 16 .
$$


We also use the inequality

$$
\sum_{k=1}^{2 p} \frac{(-1)^{k-1}}{k} t^{k}<\ln (1+t)<\sum_{k=1}^{2 p+1} \frac{(-1)^{k-1}}{k} t^{k} \quad(t>0),
$$

which easily follows from the Taylor formula.

Proof of Theorem 1. By direct verification, the required inequalities are true for $n=1,2$, so we assume $n \geq 3$. The sequence

$$
a_{n}=G_{n}-c_{0}-\frac{1}{\pi} \ln \left(n+\frac{3}{4}\right)
$$

converges to zero and it suffices to show that $\left(a_{n}\right)_{n \geq 1}$ is strictly decreasing. We have

$a_{n}-a_{n-1}=\frac{1}{16^{n}}\left(\frac{\Gamma(2 n+1)}{(\Gamma(n+1))^{2}}\right)^{2}-\frac{1}{\pi} \ln \left(1+\frac{1}{n-\frac{1}{4}}\right)<e^{v(n)}-\frac{1}{\pi} \sum_{k=1}^{4} \frac{(-1)^{k-1}}{k\left(n-\frac{1}{4}\right)^{k}}$,

so we have to prove that $f(x)<0$, where

$$
f(x)=v(x)-\ln \left(\frac{1}{\pi} \sum_{k=1}^{4} \frac{(-1)^{k-1}}{k\left(x-\frac{1}{4}\right)^{k}}\right) .
$$

We have

$$
f^{\prime}(x)=\frac{P(x)}{33792 x^{10}(4 x-1)\left(148 x-240 x^{2}+192 x^{3}-73\right)},
$$

where

$$
P(x)=5947392 x^{11}-13381632 x^{10}+\cdots-73 .
$$

The polynomial $P(x+3)$ has all coefficients positive, so $P>0$ on $[3, \infty)$. Thus $f$ is strictly increasing on $[3, \infty)$, with $f(\infty)=0$, so $f(x)<0$, for every $x \in[3, \infty)$.

The sequence

$$
b_{n}=G_{n}-c_{0}-\frac{1}{\pi} \ln \left(n+\frac{3}{4}+\frac{11}{192 n}\right)
$$

converges to zero and it suffices to show that $\left(b_{n}\right)_{n \geq 1}$ is strictly increasing. We have

$$
\begin{aligned}
b_{n}-b_{n-1} & =\frac{1}{16^{n}}\left(\frac{\Gamma(2 n+1)}{(\Gamma(n+1))^{2}}\right)^{2}-\frac{1}{\pi} \ln \left(1+\frac{1+\frac{11}{192 n}-\frac{11}{192(n-1)}}{n-\frac{1}{4}+\frac{11}{192(n-1)}}\right) \\
> & e^{u(n)}-\frac{1}{\pi} \sum_{k=1}^{5} \frac{(-1)^{k-1}}{k\left(\frac{n-\frac{1}{4}+\frac{11}{192(n-1)}}{1+\frac{11}{192 n}-\frac{11}{192(n-1)}}\right)^{k}},
\end{aligned}
$$

so we have to prove that $g(x)>0$, where

$$
g(x)=u(x)-\ln \left(\frac{1}{\pi} \sum_{k=1}^{5} \frac{(-1)^{k-1}}{k\left(\frac{x-\frac{1}{4}+\frac{11}{192(x-1)}}{1+\frac{11}{192 x}-\frac{11}{192(x-1)}}\right)^{k}}\right) .
$$

We have

$$
g^{\prime}(x)=-\frac{Q(x)}{33792 x^{10}\left(192 x^{2}-240 x+59\right)\left(192 x^{2}-192 x-11\right) R(x)}
$$


where

$Q(x)=39279662575669739520 x^{22}-273610843762924191744 x^{21}+\cdots+116760686592$ and

$R(x)=81537269760 x^{12}-448454983680 x^{11}+1085464903680 x^{10}+\cdots+175692$.

The polynomials $Q(x+3)$ and $R(x+3)$ have all coefficients positive, so $g^{\prime}<0$ on $[3, \infty)$. Thus $g$ is strictly decreasing on $[3, \infty)$, with $g(\infty)=0$, so $g(x)>0$, for every $x \in[2, \infty)$.

Proof of Theorem 2. The required inequalities are true for $n=1,2$, so we assume $n \geq 3$. The sequence

$$
\begin{aligned}
x_{n} & =c_{0}+\frac{1}{\pi} \ln (n+1)-\frac{1}{4 \pi(n+1)}+\frac{5}{192 \pi(n+1)^{2}} \\
& +\frac{3}{128 \pi(n+1)^{3}}-\frac{341}{122880 \pi(n+1)^{4}}-\frac{75}{8192 \pi(n+1)^{5}}-G_{n}
\end{aligned}
$$

converges to zero, so it suffices to prove that $\left(x_{n}\right)_{n \geq 1}$ is increasing. In this sense, we have

$$
\begin{aligned}
x_{n}-x_{n-1} & =\frac{1}{\pi} \ln \left(1+\frac{1}{n}\right)+\frac{1}{4 \pi}\left(\frac{1}{n}-\frac{1}{n+1}\right)-\frac{5}{192 \pi}\left(\frac{1}{n^{2}}-\frac{1}{(n+1)^{2}}\right) \\
& -\frac{3}{128 \pi}\left(\frac{1}{n^{3}}-\frac{1}{(n+1)^{3}}\right)+\frac{341}{122880 \pi}\left(\frac{1}{n^{4}}-\frac{1}{(n+1)^{4}}\right) \\
& +\frac{75}{8192 \pi}\left(\frac{1}{n^{5}}-\frac{1}{(n+1)^{5}}\right)-\frac{1}{16^{n}}\left(\frac{\Gamma(2 n+1)}{(\Gamma(n+1))^{2}}\right)^{2} .
\end{aligned}
$$

Using

$$
\ln \left(1+\frac{1}{n}\right)>\sum_{k=1}^{10} \frac{(-1)^{k-1}}{k n^{k}}
$$

and (2.3), we get

$$
x_{n}-x_{n-1}>S(n)-\exp v(n),
$$

where

$$
\begin{aligned}
S(n) & =\frac{1}{\pi} \sum_{k=1}^{10} \frac{(-1)^{k-1}}{k n^{k}}+\frac{1}{4 \pi}\left(\frac{1}{n}-\frac{1}{n+1}\right)-\frac{5}{192 \pi}\left(\frac{1}{n^{2}}-\frac{1}{(n+1)^{2}}\right) \\
& -\frac{3}{128 \pi}\left(\frac{1}{n^{3}}-\frac{1}{(n+1)^{3}}\right)+\frac{341}{122880 \pi}\left(\frac{1}{n^{4}}-\frac{1}{(n+1)^{4}}\right) \\
& +\frac{75}{8192 \pi}\left(\frac{1}{n^{5}}-\frac{1}{(n+1)^{5}}\right) .
\end{aligned}
$$

Following (2.4), we have to prove that $h(x)>0$, for every $x \geq 3$, where

$$
h(x)=\ln S(x)-v(x) .
$$

We have

$$
h^{\prime}(x)=-\frac{W(x)}{33792 x^{10}(x+1) T(x)},
$$


where

$$
W(x)=2894918400 x^{18}+39555584640 x^{17}+\cdots+258048
$$

and

$$
T(x)=2580480 x^{14}+12257280 x^{13}+\cdots-258048 .
$$

The polynomials $W(x+3)$ and $T(x+3)$ have all coefficients positive, so $W>0$ and $T>0$, on $[3, \infty)$. In consequence, $h^{\prime}<0$, so $h$ is strictly decreasing on $[3, \infty)$. But $h(\infty)=0$, thus $h(x)>0$, for every $x \in[3, \infty)$.

The sequence

$$
\begin{aligned}
y_{n} & =c_{0}+\frac{1}{\pi} \ln (n+1)-\frac{1}{4 \pi(n+1)}+\frac{5}{192 \pi(n+1)^{2}} \\
& +\frac{3}{128 \pi(n+1)^{3}}-\frac{341}{122880 \pi(n+1)^{4}}-G_{n}
\end{aligned}
$$

converges to zero, so it suffices to prove that $\left(y_{n}\right)_{n \geq 1}$ is strictly decreasing. In this sense, we have

$$
\begin{gathered}
y_{n}-y_{n-1}=\frac{1}{\pi} \ln \left(1+\frac{1}{n}\right)+\frac{1}{4 \pi}\left(\frac{1}{n}-\frac{1}{n+1}\right)-\frac{5}{192 \pi}\left(\frac{1}{n^{2}}-\frac{1}{(n+1)^{2}}\right) \\
-\frac{3}{128 \pi}\left(\frac{1}{n^{3}}-\frac{1}{(n+1)^{3}}\right)+\frac{341}{122880 \pi}\left(\frac{1}{n^{4}}-\frac{1}{(n+1)^{4}}\right)-\frac{1}{16^{n}}\left(\frac{\Gamma(2 n+1)}{(\Gamma(n+1))^{2}}\right)^{2} .
\end{gathered}
$$

Using

$$
\ln \left(1+\frac{1}{n}\right)<\sum_{k=1}^{9} \frac{(-1)^{k-1}}{k n^{k}}
$$

and (2.3), we get

$$
y_{n}-y_{n-1}<Z(n)-\exp u(n),
$$

where

$$
\begin{aligned}
Z(n) & =\frac{1}{\pi} \sum_{k=1}^{9} \frac{(-1)^{k-1}}{k n^{k}}+\frac{1}{4 \pi}\left(\frac{1}{n}-\frac{1}{n+1}\right)-\frac{5}{192 \pi}\left(\frac{1}{n^{2}}-\frac{1}{(n+1)^{2}}\right) \\
& -\frac{3}{128 \pi}\left(\frac{1}{n^{3}}-\frac{1}{(n+1)^{3}}\right)+\frac{341}{122880 \pi}\left(\frac{1}{n^{4}}-\frac{1}{(n+1)^{4}}\right) .
\end{aligned}
$$

Following (2.5), we have to prove that $j(x)<0$, for every $x>0$, where

$$
j(x)=\ln Z(x)-u(x) .
$$

We have

$$
j^{\prime}(x)=\frac{M(x)}{33792 x^{10}(x+1) N(x)},
$$

where

$$
M(x)=19958400000 x^{17}+26044761600 x^{16}+\cdots-293601280
$$

and

$$
N(x)=2580480 x^{12}+9676800 x^{11}+\cdots+286720 .
$$

As all the coefficients of the polynomials $M(x+3)$ and $N(x+3)$ are positive, it results that $M>0$ and $N>0$ on $[3, \infty)$. In consequence, $j^{\prime}>0$. Finally, $j<0$, on $[3, \infty)$, since $j$ is strictly increasing, with $j(\infty)=0$. 
Proof of Theorem 3. The required inequalities are true for $n=1,2$ and we assume that $n \geq 3$. We prove that the sequence

$$
t_{n}=G_{n}-c_{0}-\frac{1}{\pi} \psi\left(n+\frac{5}{4}\right)-\frac{1}{64 \pi n^{2}}+\frac{3}{128 \pi n^{3}}
$$

is strictly decreasing. In this sense,

$$
\begin{aligned}
t_{n}-t_{n-1} & =\frac{1}{16^{n}} \frac{(\Gamma(2 n+1))^{2}}{(\Gamma(n+1))^{4}}-\frac{1}{\pi\left(n+\frac{1}{4}\right)} \\
& -\frac{1}{64 \pi n^{2}}+\frac{3}{128 \pi n^{3}}+\frac{1}{64 \pi(n-1)^{2}}-\frac{3}{128 \pi(n-1)^{3}}
\end{aligned}
$$

and we have to prove that $m<0$, where

$m(x)=v(x)-\ln \left(\frac{1}{\pi\left(x+\frac{1}{4}\right)}+\frac{1}{64 \pi x^{2}}-\frac{3}{128 \pi x^{3}}-\frac{1}{64 \pi(x-1)^{2}}+\frac{3}{128 \pi(x-1)^{3}}\right)$.

We have

$$
m^{\prime}(x)=\frac{L(x)}{33792 x^{10}(x-1)(4 x+1)\left(x-29 x^{2}-456 x^{3}+1520 x^{4}-1536 x^{5}+512 x^{6}+3\right)},
$$

where

$$
L(x)=23384064 x^{13}-25681920 x^{12}+\cdots+3 .
$$

As the polynomials involved in the expression of $m^{\prime}$ have all coefficients positive when we replace $x$ by $x+3$, it follows that $m^{\prime}>0$ on $[3, \infty)$. In consequence, $m$ is strictly increasing with $m(\infty)=0$, so $m<0$.

Now we prove that the sequence

$$
z_{n}=G_{n}-c_{0}-\frac{1}{\pi} \psi\left(n+\frac{5}{4}\right)-\frac{1}{64 \pi n^{2}}+\frac{3}{128 \pi n^{3}}-\frac{173}{8192 \pi n^{4}}
$$

is strictly increasing. In this sense,

$$
\begin{aligned}
z_{n}-z_{n-1} & =\frac{1}{16^{n}} \frac{(\Gamma(2 n+1))^{2}}{(\Gamma(n+1))^{4}}-\frac{1}{\pi\left(n+\frac{1}{4}\right)} \\
& -\frac{1}{64 \pi n^{2}}+\frac{3}{128 \pi n^{3}}-\frac{173}{8192 \pi n^{4}}+\frac{1}{64 \pi(n-1)^{2}} \\
& -\frac{3}{128 \pi(n-1)^{3}}+\frac{173}{8192 \pi(n-1)^{4}},
\end{aligned}
$$

and we have to prove that $n(x)>0$, where

$$
\begin{aligned}
& n(x)=u(x)-\ln \left(\frac{1}{\pi\left(x+\frac{1}{4}\right)}+\frac{1}{64 \pi x^{2}}-\frac{3}{128 \pi x^{3}}+\frac{173}{8192 \pi x^{4}}\right. \\
&\left.\quad-\frac{1}{64 \pi(x-1)^{2}}+\frac{3}{128 \pi(x-1)^{3}}-\frac{173}{8192 \pi(x-1)^{4}}\right) .
\end{aligned}
$$

We have

where

$$
n^{\prime}(x)=-\frac{K(x)}{33792 x^{10}(x-1)(4 x+1) J(x)},
$$

$$
K(x)=1175961600 x^{14}-845070336 x^{13}+\cdots+177152
$$


and

$$
\begin{aligned}
J(x) & =5380 x^{3}-1602 x^{2}-192 x+24560 x^{4}-126464 x^{5} \\
& +195584 x^{6}-131072 x^{7}+32768 x^{8}+173 .
\end{aligned}
$$

But the polynomials $K(x+3)$ and $J(x+3)$ have all coefficients positive, so $n^{\prime}<0$ on $[3, \infty)$. Finally, $n(x)>0$, for every $x \in[3, \infty)$, since it is strictly decreasing with $n(\infty)=0$.

\section{ACKNOWLEDGEMENT}

The author thanks the anonymous referees for their useful comments and ideas which improved the initial form of this paper.

\section{REFERENCES}

[1] H. Alzer, On some inequalities for the gamma and psi functions, Math. Comp., 66 (1997), no. 217, 373-389. MR.1388887 (97e:33004)

[2] H. Alzer, Inequalities for the constants of Landau and Lebesgue, J. Comput. Appl. Math., 139 (2002) 215-230. MR.1876636 (2002j:26013)

[3] L. Brutman, A sharp estimate of the Landau constants, J. Approx. Theory, 34 (1982), 217-220. MR654287 (84m:41058)

[4] D. Cvijović, J. Klinowski, Inequalities for the Landau constants, Math. Slovaca, 50 (2000), 159-164. MR 1763118 (2001e:11125)

[5] L.P. Falaleev, Inequalities for the Landau constants, Siberian Math. J. 32 (1991), 896-897. MR.1155818 (93c:30001)

[6] E. Landau, Abschätzung der Koeffzientensumme einer Potenzreihe, Arch. Math. Phys. 21 (1913), 42-50, 250-255.

[7] G.N. Watson, The constants of Landau and Lebesgue, Quart. J. Math. Oxford Ser. 1 (2) (1930), 310-318.

[8] D. Zhao, Some sharp estimates of the constants of Landau and Lebesgue, J. Math. Anal. Appl. 349 (2009), 68-73. MR 2455731(2009h:33003)

Department of Mathematics, Valahia University of TÂrgovişte, Bd. Uniri 18, 130082 TÂrgovişte, Romania

E-mail address: cmortici@valahia.ro

$U R L:$ http://www.cristinelmortici.ro 